\title{
Conversion of the Barotropic Tide
}

\author{
Stefan G. Llewellyn SMith \\ Department of Mechanical and Aerospace Engineering, University of California, San Diego, La Jolla, California \\ W. R. YOunG \\ Scripps Institution of Oceanography, University of California, San Diego, La Jolla, California
}

(Manuscript received 11 July 2001, in final form 24 October 2001)

\begin{abstract}
Using linear wave theory, the rate at which energy is converted into internal gravity waves by the interaction of the barotropic tide with topography in an ocean is calculated. Bell's formula for the conversion rate is extended to the case of an ocean of finite depth $H$ with weak two-dimensional topography $h(x, y)$ and arbitrary buoyancy frequency $N(z)$. Approximate solutions are computed using the WKB method, which reduce to the previous result for an ocean of infinite depth with constant stratification. The conversion rate for a finite-depth ocean can be substantially smaller than the infinite-ocean prediction when the length scale of the topography is of the same order as the horizontal wavelength of the internal tide. The conversion rate for two-dimensional Gaussian seamounts is calculated. Using observed statistics for the distribution of seamounts, the authors estimate 1/4 $\mathrm{GW}$ of conversion for a square of ocean floor of side $1000 \mathrm{~km}$.
\end{abstract}

\section{Introduction}

The tidal sloshing of a stratified ocean over a bumpy bottom transfers energy from the spin of the earth into internal gravity waves. The resulting gravity waves are known as the "internal tide" [for a review, see Wunsch (1975)]. This conversion radiatively damps the barotropic tide. The tidal-conversion process, illustrated in Fig. 1, may make an important contribution to mixing the deep ocean and catalyzing the thermohaline circulation (Munk and Wunsch 1998). St. Laurent and Garrett (2001, submitted to J. Phys. Oceanogr.) discuss the processes that might degrade the internal tide into smallscale mixing.

Following the early papers of Rattray (1960), Cox and Sandstrom (1962), Baines (1973), and Bell (1975a,b), the problem of the internal tide received only episodic attention. But the observations of Ledwell et al. (2000) of elevated mixing rates over rough topography, and the global tidal analysis of Egbert and Ray (2000), have sparked recent interest in the role of the internal tide in ocean mixing. Numerical investigations have used both idealized (Holloway and Merrifield 1999; Khatiwala 2001, submitted to Deep-Sea Res.; Li 2001, submitted to J. Mar Res.) and realistic (Kang et al. 2000; Cummins et al. 2001; Merrifield and Holloway

Corresponding author address: Dr. Stefan G. Llewellyn Smith, Dept. of Mechanical and Aerospace Engineering, University of California, San Diego, 9500 Gilman Drive, La Jolla, CA 92093-0411. E-mail: sgls@ucsd.edu
2001, manuscript submitted to J. Geophys. Res.) topography.

The most useful analytic estimate of the conversion rate is that of Bell (1975a), which assumes inter alia (i) that the "fluid has unlimited vertical extent," (ii) that the topography varies only in one direction $(x)$, and (iii) that the buoyancy frequency $N$ is uniform. Bell (1975b) removed the second restriction, and gave an estimate for tidal conversion by two-dimensional topography. Our goal is to remove all of these restrictions and calculate the conversion rate associated with two-dimensional topography, $h(x, y)$, in an ocean of finite depth with buoyancy frequency $N(z)$. However we do continue to follow Bell in assuming that the topographic slopes are much less than the slope of a tidal beam and that the topographic height is much less than both the vertical wavelength of the internal tide and the depth of the ocean. This small amplitude assumption means that the bottom boundary condition can be applied approximately at a flat surface, $z=-H$, rather than at the actual bottom $z=-H+h$.

The barotropic tidal velocity is represented as

$$
\mathbf{U}=\left[U_{0} \cos \left(\omega_{0} t\right), V_{0} \cos \left(\omega_{0} t+\chi\right)\right] .
$$

Bell's (1975a) formula for the conversion rate with infinite depth and $h_{y}=0$ is

$$
C_{\text {Bell }}=\rho_{0} U_{0}^{2} L N_{B} \sqrt{1-\frac{f_{0}^{2}}{\omega_{0}^{2}}} \int_{0}^{\infty} k \tilde{h}(k) \tilde{h}^{*}(k) \frac{d k}{2 \pi} \text { (watts). }
$$




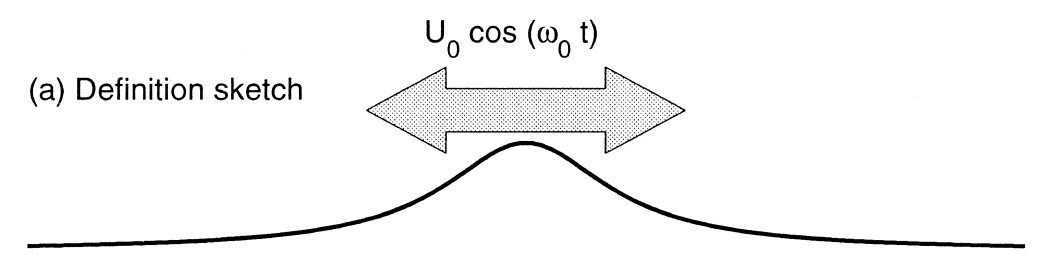

(b) Tidal conversion by a ridge

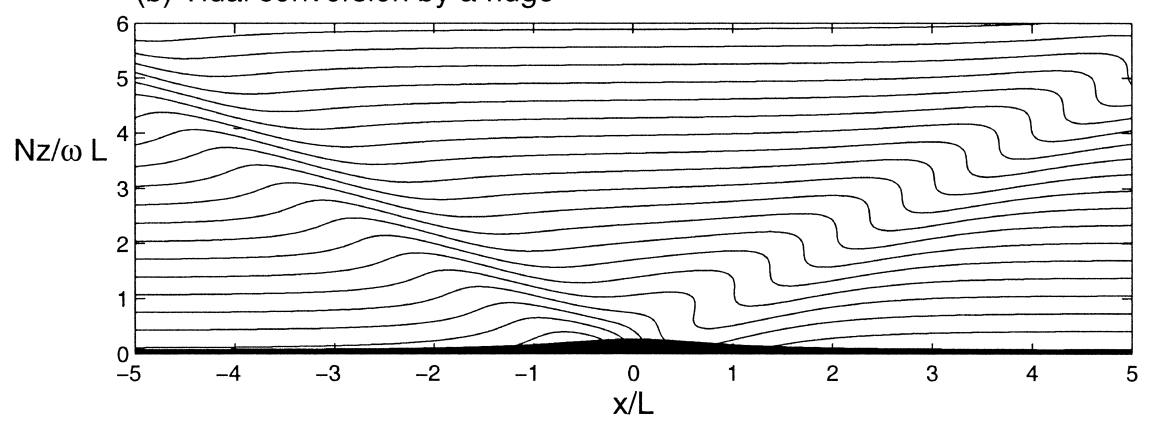

FIG. 1. The periodically reversing barotropic tide in (1) flows over a bottom bump and radiates gravity waves into a stably stratified ocean with buoyancy frequency $N$. The illustration above is obtained using Bell's (1975a) solution.

In (2), $N_{B} \equiv N(-H)$ is the buoyancy frequency at the bottom of the ocean, $L$ is the length (in $y$ ) of the topography, and

$$
\tilde{h}(k) \equiv \int_{-\infty}^{\infty} e^{-i k x} h(x) d x
$$

is the Fourier transform of $h(x)$. Because the topography does not vary in $y$, the alongridge component of the tidal velocity, $V_{0} \cos \left(\omega_{0} t+\chi\right)$, is irrelevant.

In (2) we have further simplified Bell's equation (5.6) with two additional approximations. First, we have made the hydrostatic approximation (and we have included the effects of rotation, $f_{0} \neq 0$ ). Second, we have taken Bell's "quasistatic limit" so that the dominant contribution to the conversion is into the fundamental tidal frequency, $\omega_{0}$. This approximation is discussed in more detail in (14) below. Finally, by using $N_{B}$ in (2), we have anticipated one of our results that with nonuniform stratification, $N(z)$, the best approximation (in the WKB sense) is obtained by using $N_{B}$ in Bell's results. The conversion rate $\mathcal{C}_{\text {Bell }}$ in (2) provides the simplest estimate of the tidal conversion: we will compare our more elaborate estimates with $\mathcal{C}_{\text {Bell }}$.

To highlight some recent estimates of tidal conversion, we evaluate $\mathcal{C}_{\text {Bell }}$ using numbers that very roughly correspond to the Hawaiian Ridge. The ridge is represented as a witch of Agnesi (Bell 1975a),

$$
h(x)=\frac{h_{\max }}{1+\mu^{2} x^{2}},
$$

where $l \equiv 1 / \mu$ is the width of the ridge. Then, evaluating the Fourier transform $\tilde{h}(k)$, we find from (2) that

$$
C_{\text {Bell }}=\frac{\pi}{8} \rho_{0} U_{0}^{2} L N_{B} h_{\max }^{2} \sqrt{1-\frac{f_{0}^{2}}{\omega_{0}^{2}}} .
$$

There is a small surprise: $\mathcal{C}_{\text {Bell }}$ is independent of the width $l$ of the ridge. For the length of the ridge we take $L=1000 \mathrm{~km}$ and for the height $h_{\max }=4 \mathrm{~km}$. Further, take $f_{0}=10^{-4} \mathrm{~s}^{-1}$ and $N_{B}=10 f_{0}$ (i.e., $0.57 \mathrm{cph}$ ). For the $M_{2}$ tide, take $U_{0}=4 \times 10^{-2} \mathrm{~m} \mathrm{~s}^{-1}$ and $\omega_{0}=2 f_{0}$.

With $h_{\max }=4 \mathrm{~km}$ we are recklessly extrapolating results based on the weak topography approximation (15) far outside its range of validity. A tidal velocity of $4 \mathrm{~cm} \mathrm{~s}^{-1}$ is typical of flow through shallow Hawaiian passages. A cautious reader can make appropriate reductions by noticing that $\mathcal{C}_{\text {Bell }} \propto h_{\max }^{2} U_{0}^{2}$. In any event, using the numbers in the previous paragraph,

$$
\mathcal{C}_{\mathrm{Bell}}=8.6 \mathrm{GW} \text {. }
$$

This is the same order of magnitude as the $9.7 \mathrm{GW}$ of internal wave energy radiated from the Hawaiian Ridge estimated by Merrifield and Holloway (2001) using the Princeton Ocean Model. Using a two-layer model, Kang et al. (2000) estimate $5.4 \mathrm{GW}$ of $M_{2}$ conversion into the first baroclinic mode at Hawaii. And, with TOPEX/ Poseidon altimetry, Ray and Mitchum (1996) estimated $15 \mathrm{GW}$ of conversion into the first baroclinic mode at the Hawaiian Ridge. Finally, Munk (1996) estimated that $200 \mathrm{GW}$ of $M_{2}$ conversion occurs globally along $50000 \mathrm{~km}$ of submarine ridges.

In section 2 we formulate the tidal conversion problem and discuss the assumptions necessary to obtain a tractable linear problem. In section 3 we solve this linear problem by first projecting onto the vertical normal modes of the stratification $N(z)$; each modal amplitude 
satisfies the forced shallow water equations. We obtain a solution of this system using a Green's function. Fortunately this integral representation is only a bridge: the conversion into mode $n$, denoted by $\mathcal{C}_{n}$, can be calculated without evaluating convolution integrals. This evaluation gives the main result of this paper: formula (37). In section 4 we discuss approximations and reductions of (37). In section 5 we calculate the tidal conversion produced by a one-dimensional ridge in an ocean with finite depth $H$. We show that $\mathcal{C}_{\text {Bell }}$ in (5) is accurate to about $10 \%$ provided that

$$
\alpha=\frac{1}{\sqrt{\left(\omega_{0} / f_{0}\right)^{2}-1}} \frac{\lambda_{0}}{l}
$$

is greater than about 2 . [ $\operatorname{In}(7), \lambda_{1}$ is the radius of deformation of the first baroclinic mode.] The effect of finite depth is always to reduce $C$ below the estimate $C_{\text {Bell }}$ and, if $\alpha$ is small, the converted energy is concentrated into low vertical modes. In section 6 we calculate conversion rates for both axisymmetric and anisotropic Gaussian topography. Section 7 is the conclusion that includes an estimate for the global tidal conversion produced by seamounts.

\section{Formulation}

Consider an ocean of nonuniform finite depth with the surface at $z=0$ so that $-H+h(x, y)<z<0$. Here $H$ is a constant and $h(x, y)$ is the bumpy bottom. The density is written as

$$
\rho=\rho_{0}\left(1+g^{-1} \int_{z}^{0} N^{2}\left(z^{\prime}\right) d z^{\prime}-g^{-1} b\right),
$$

where $b(x, y, t)$ is the buoyancy of the wavy disturbance and $N(z)$ is the buoyancy frequency.

\section{a. The problem}

The problem is to solve

$$
\begin{array}{rlrl}
u_{t}-f_{0} v+p_{x} & =0, & v_{t}+f_{0} u+p_{y}=0, \\
p_{z} & =b, & b_{t}+w N^{2}=0, \\
u_{x}+v_{y}+w_{z} & =0, & &
\end{array}
$$

with the boundary conditions

$$
w(x, y, 0, t)=0, \quad w(x, y,-H, t)=\mathbf{U} \cdot \boldsymbol{\nabla} h ;
$$

$\mathbf{U}(t)$ in the bottom boundary condition is the barotropic tidal velocity in (1). In addition to the bottom boundary condition, there is a radiation condition that as $|\mathbf{x}| \rightarrow$ $\pm \infty$ there is only outward going energy. We will be more specific about implementing this later.

\section{b. Energetics}

The energy conservation equation is

$$
E_{t}+\boldsymbol{\nabla} \cdot \mathbf{J}=0
$$

where the density $E$ and flux $\mathbf{J}$ are

$E \equiv \frac{1}{2} \rho_{0}\left(u^{2}+v^{2}+N^{-2} b^{2}\right), \quad \mathbf{J}=\rho_{0} p(u, v, w)$.

The object of our desire is the conversion rate

$$
\mathcal{C} \equiv \rho_{0} \int\left\langle p_{B} \mathbf{U}\right\rangle \nabla \cdot h d^{2} \mathbf{x},
$$

where $p_{B} \equiv p(x, y,-H, t)$ is the bottom pressure and angle brackets denote a phase average.

\section{c. Three approximations}

Three approximations have been made to obtain the tractable linear problem in (9) and (10). First, because $\omega_{0} / N \ll 1$, we use the hydrostatic approximation. Second, we assume that the tidal excursion distance, $U_{0} /$ $\omega_{0}$, is much less than the horizontal scale of the topography $l$ :

$$
U_{0} /\left(\omega_{0} l\right) \ll 1 .
$$

Inequality (14) ensures that the problem is linear and that the advective effects of the barotropic tide are negligible; that is, terms such as $U b_{x}$ are much smaller than $b_{t}$. Bell (1975a) does not make this approximation and this is why Bell's calculation contains all harmonics of the tide such as $\pm 2 \omega_{0}$ and so on. The simplifying assumption in (14) is realistic. For instance, with a deep tidal velocity $U_{0}=4 \mathrm{~cm} \mathrm{~s}^{-1}$, the excursion of the $M_{2}$ tide is only $280 \mathrm{~m}$, which is small relative to nearly all topographic features of interest.

The third and most problematic approximation in (9) and (10) is that the topography is weak: this means that the bottom boundary condition is applied at the flat surface $z=-H$ rather than at the actual position of the bottom boundary $z=-H+h$. This weak topography approximation is justified provided that

$$
\frac{\text { amplitude of the topography }}{\text { vertical scale of the waves }} \ll 1
$$

and that topographic slopes are much less than the slope of a tidal beam. These weak-topography approximations can fail for realistic topographic features. For instance, suppose that $k^{-1}=l=10 \mathrm{~km}, \omega_{0}=2 f_{0}$, and $N=$ $20 f_{0}$. Using the internal wave dispersion relation

$$
m^{-1}=k^{-1} \frac{\sqrt{\omega_{0}^{2}-f_{0}^{2}}}{N},
$$

we estimate that the vertical scale of the internal tide, $\mathrm{m}^{-1}$, is $870 \mathrm{~m}$. Bathymetric excursions of this size can occur over distances of $10 \mathrm{~km}$ so the parameter in (15) could be of order unity. 


\section{Solution}

a. Vertical modes

We begin by projecting (9) and (10) onto the vertical modes associated with the stratification $N(z)$. These functions are defined by the eigenproblem

$\frac{d^{2} a_{n}}{d z^{2}}+c_{n}^{-2} N^{2} a_{n}=0, \quad a_{n}(0)=a_{n}(-H)=0$,

where the eigenvalue $c_{n}$ is the phase speed of mode $n$ and

$$
\lambda_{n} \equiv \frac{c_{n}}{f_{0}}
$$

is the radius of deformation of mode $n$. In addition to $a_{n}(z)$ we need the functions

$$
a_{n}^{\prime}(z) \equiv \frac{d a_{n}}{d z}
$$

which satisfy

$$
\begin{aligned}
\frac{d}{d z}\left(N^{-2} \frac{d a_{n}^{\prime}}{d z}\right)+c_{n}^{-2} a_{n}^{\prime} & =0, \\
\frac{d a_{n}^{\prime}}{d z}(0) & =\frac{d a_{n}^{\prime}}{d z}(-H)=0 .
\end{aligned}
$$

We take $a_{n}$ to be dimensionless so that $a_{n}^{\prime}$ has dimensions (length $)^{-1}$. The orthogonality conditions are

$$
\begin{aligned}
\int_{-H}^{0} a_{n}(z) a_{m}(z) N^{2}(z) d z & =c_{n}^{2} \int_{-H}^{0} a_{n}^{\prime}(z) a_{m}^{\prime}(z) d z \\
& =\lambda_{n} f_{0}^{2} \varepsilon_{n} \delta_{m n},
\end{aligned}
$$

where $\varepsilon_{n}$ is a dimensionless normalization constant associated with mode $n$.

\section{b. Modal representation of the solution}

The solution is represented as

$$
\begin{aligned}
(u, v, p) & =\sum_{n=1}^{\infty} \lambda_{n}\left(u_{n}, v_{n}, p_{n}\right) a_{n}^{\prime}(z) \quad \text { and } \\
\left(w, f_{0}^{2} N^{-2} b\right) & =\sum_{n=1}^{\infty}\left(w_{n}, b_{n}\right) a_{n}(z) .
\end{aligned}
$$

The factors $\lambda_{n}$ are included in these definitions so that $u_{n}$ has the same dimensions as $u$ and similarly for the other quantities. The vertically integrated energy density is

$$
\begin{aligned}
& \frac{1}{2} \rho_{0} \int_{-H}^{0}\left(u^{2}+v^{2}+N^{-2} b^{2}\right) d z \\
& \quad=\frac{1}{2} \rho_{0} \sum_{n=1}^{\infty} \lambda_{n} \varepsilon_{n}\left(u_{n}^{2}+v_{n}^{2}+f_{0}^{-2} b_{n}^{2}\right) .
\end{aligned}
$$

We project the equations of motion onto the vertical normal modes by multiplying $(9 \mathrm{a}, \mathrm{b}, \mathrm{e})$ by $a_{m}^{\prime}$ and $(9 \mathrm{c}, \mathrm{d})$ by $a_{m}$. We then integrate over $(-H, 0)$ and uses modal orthogonality. Because one cannot exchange differentiation and summation in the series (23) for $w, a_{m}^{\prime} w_{z}$ in the continuity equation $(9 \mathrm{e})$ is integrated by parts in the usual Galerkin fashion. The term that falls outside the integrals leads to a forcing function proportional to $w(x$, $y,-H, t)$ in the modal equations. We find the following forced shallow-water equations for the modal amplitudes

$$
\begin{aligned}
u_{n t}-f_{0} v_{n}+p_{n x} & =0, \quad v_{n t}+f_{0} u_{n}+p_{n y}=0, \\
p_{n}+\lambda_{n} b_{n} & =0, \quad b_{n t}+f_{0}^{2} w_{n}=0, \\
p_{n t}+c_{n}^{2}\left(u_{n x}+v_{n y}\right) & =f_{0}^{2} \lambda_{n} \varsigma_{n} \mathbf{U} \cdot \boldsymbol{\nabla} h,
\end{aligned}
$$

where the dimensionless constant $\varsigma_{n}$ is

$$
\varsigma_{n} \equiv \varepsilon_{n}^{-1} \lambda_{n} a_{n}^{\prime}(-H) .
$$

The modal equations (25) have a conservation law

$$
\begin{aligned}
& \frac{1}{2}\left[u_{n}^{2}+v_{n}^{2}+c_{n}^{-2} p_{n}^{2}\right]_{t}+\nabla \cdot\left[\left(u_{n}, v_{n}\right) p_{n}\right] \\
& \quad=\varsigma_{n} \lambda_{n}^{-1} p_{n} \mathbf{U} \cdot \boldsymbol{\nabla} h .
\end{aligned}
$$

Comparing (27) with (24), we identify the modal conversion rate as

$$
\mathcal{C}_{n}=\rho_{0} \varepsilon_{n} \boldsymbol{\varsigma}_{n} \int\left\langle p_{n} \mathbf{U}\right\rangle \cdot \nabla h d^{2} \mathbf{x} \quad \text { (watts). }
$$

That is, the total conversion is simply

$$
\mathcal{C}=\sum_{n=1}^{\infty} \mathcal{C}_{n}
$$

\section{c. The Klein-Gordon equation and its solution}

With some algebra we can reduce (25) to a forced Klein-Gordon equation

$\left(\partial_{t}^{2}+f_{0}^{2}\right) p_{n t}-c_{n}^{2} \nabla^{2} p_{n t}=f_{0}^{2} \lambda_{n} \varsigma_{n}\left(\partial_{t}^{2}+f_{0}^{2}\right) \mathbf{U} \cdot \boldsymbol{\nabla} h$.

Next, we write

$$
\begin{aligned}
p_{n} & =\hat{p}_{n} e^{-i \omega_{0} t}+\hat{p}_{n}^{*} e^{i \omega_{0} t} \quad \text { and } \\
\mathbf{U} & =\hat{\mathbf{U}} e^{-i \omega_{0} t}+\hat{\mathbf{U}} e^{i \omega_{0} t} .
\end{aligned}
$$

Notice that from (1), $\hat{\mathbf{U}}=1 / 2\left[U_{0}, e^{-i \chi} V_{0}\right]$. Putting (31) and (32) into (30) gives

$$
\nabla^{2} \hat{p}_{n}+\kappa_{n}^{2} \hat{p}_{n}=i \kappa_{n} \boldsymbol{\varsigma}_{n} f_{0} \sqrt{1-\frac{f_{0}^{2}}{\omega_{0}^{2}}} \hat{\mathbf{U}} \cdot \nabla h,
$$

where

$$
\kappa_{n} \equiv \frac{\sqrt{\omega_{0}^{2}-f_{0}^{2}}}{c_{n}}=\frac{\omega_{0}}{f_{0}} \sqrt{1-\frac{f_{0}^{2}}{\omega_{0}^{2}}} \frac{1}{\lambda_{n}} .
$$

Equation (33) is solved using a Green's function 


$$
\hat{p}_{n}(\mathbf{x})=i \kappa_{n} \varsigma_{n} f_{0} \sqrt{1-\frac{f_{0}^{2}}{\omega_{0}^{2}}} \hat{\mathbf{U}} \cdot \int \mathcal{G}\left(\kappa_{n} s\right) \boldsymbol{\nabla}^{\prime} h\left(\mathbf{x}^{\prime}\right) d^{2} \mathbf{x}^{\prime},
$$

where $\mathcal{G}(\zeta) \equiv\left[Y_{0}(\zeta)-i J_{0}(\zeta)\right] / 4$ and $s \equiv\left|\mathbf{x}-\mathbf{x}^{\prime}\right|$. We use the combination $J_{0}+i Y_{0}$ in the Green's function to take care of the radiation condition.

Putting (35) into the expression for $\mathcal{C}_{n}$ in (28) gives

$C_{n}=\frac{1}{8} \rho_{0} f_{0} \sqrt{1-\frac{f_{0}^{2}}{\omega_{0}^{2}}} \kappa_{n} \varepsilon_{n} \varsigma_{n}^{2} \iint J_{0}\left(\kappa_{n} s\right)\left[U_{0}^{2} \partial_{x} \partial_{x^{\prime}}+U_{0} V_{0} \cos \chi\left(\partial_{x} \partial_{y^{\prime}}+\partial_{x^{\prime}} \partial_{y}\right)+V_{0}^{2} \partial_{y} \partial_{y^{\prime}}\right] h(\mathbf{x}) h\left(\mathbf{x}^{\prime}\right) d^{2} \mathbf{x} d^{2} \mathbf{x}^{\prime}$

The result above entails a fourfold integration over both $\mathbf{x}=(x, y)$ and $\mathbf{x}^{\prime}=\left(x^{\prime}, y^{\prime}\right)$. However the convolution involving $J_{0}\left(\kappa_{n} s\right)$ indicates that we can simplify these integrals by going into Fourier space. The result is particularly simple because the Fourier transform $(r \rightarrow \kappa)$ takes $J_{0}\left(\kappa_{n} r\right) \rightarrow 2 \pi \delta\left(\kappa-\kappa_{n}\right) / \kappa_{n}$. This $\delta\left(\kappa-\kappa_{n}\right)$ enables integration in the Fourier space.

Making the simplifications outlined above, (36) becomes

$$
\begin{aligned}
C_{n}= & \frac{1}{16} \rho_{0} f_{0}\left(U_{0}^{2}+V_{0}^{2}\right) \sqrt{1-\frac{f_{0}^{2}}{\omega_{0}^{2}}} \varepsilon_{n} \varsigma_{n}^{2} \kappa_{n}^{2} \\
& \times \oint \Xi(\phi) \mathcal{T}\left(\kappa_{n}, \phi\right) d \phi \quad \text { (watts), }
\end{aligned}
$$

where $\varepsilon_{n}, \varsigma_{n}$, and $\kappa_{n}$ are defined in (21), (26), and (34), respectively. Further in (37),

$$
\mathcal{T}(\kappa, \phi) \equiv \frac{\kappa}{2 \pi}|\tilde{h}(k, l)|^{2},
$$

where the two-dimensional Fourier transform is

$$
\tilde{h}(k, l) \equiv \iint e^{-i k x-i l y} h(x, y) d x d y,
$$

and $(k, l)=\kappa(\cos \phi$, $\sin \phi)$. The tidal ellipse in (1) affects $\mathcal{C}_{n}$ in (37) through the function

$\Xi(\phi) \equiv 1+\frac{U_{0}^{2}-V_{0}^{2}}{U_{0}^{2}+V_{0}^{2}} \cos 2 \phi+\frac{2 U_{0}^{2} \cos \chi}{U_{0}^{2}+V_{0}^{2}} \sin 2 \phi$.

Notice that Parseval's theorem is

$$
\begin{aligned}
\iint \tilde{h}^{2}(x, y) d x d y & =\iint|h(k, l)|^{2} \frac{d k d l}{(2 \pi)^{2}} \\
& =\int_{0}^{\infty} \oint \mathcal{T}(\kappa, \phi) d \kappa \frac{d \phi}{2 \pi}
\end{aligned}
$$

This equality implies that in the case of isotropic random topography, the ensemble average of $\mathcal{T}$ equals the total area of the domain times the power spectral density of the topography.

\section{Special cases and approximations}

Equation (37) for the conversion rate into vertical mode number $n$ is the most important result in this paper. The buoyancy profile $N(z)$ determines the wavenumber $\kappa_{n}$ in (34) and also the two dimensionless constants $\varepsilon_{n}$ and $\boldsymbol{s}_{n}$. Using the WKB approximation for the vertical normal modes we can obtain approximate expressions for these three quantities and rewrite (37) in a form that can be more easily interpreted in physical terms.

\section{a. The WKB approximation}

The WKB approximation is most accurate for the higher modes, but it is not misleading even for the first mode. Further, if $N_{z}=0$, then the WKB formulas below are exact for all modes. In any event, the approximation is

$$
a_{n}(z) \approx \sqrt{\frac{\bar{N}}{N(z)}} \sin \left(\frac{n \pi}{H \bar{N}} \int_{-H}^{z} N\left(z^{\prime}\right) d z^{\prime}\right),
$$

where the average buoyancy frequency is $\bar{N} \equiv H^{-1}$ $\int_{-H}^{0} N\left(z^{\prime}\right) d z^{\prime}$ and the eigenvalues are $c_{n}=f_{0} \lambda_{n} \approx \bar{N} H /$ $n \pi$ so that

$$
\kappa_{n} \approx \sqrt{\omega_{0}^{2}-f_{0}^{2}} \frac{n \pi}{\bar{N} H} .
$$

The dimensionless constants are

$$
\varepsilon_{n} \approx \frac{n \pi}{2} \frac{\bar{N}}{f_{0}}, \quad \varsigma_{n} \approx \frac{2}{n \pi} \sqrt{\frac{N_{B}}{\bar{N}}},
$$

where $N_{B} \equiv N(-H)$ is the buoyancy frequency at the bottom.

Putting these approximations into (37) gives the WKB approximation to the conversion rate

$$
\begin{aligned}
\mathcal{C}_{n} \approx & \frac{\rho_{0}}{8 \pi} N_{B}\left(U_{0}^{2}+V_{0}^{2}\right) \sqrt{1-\frac{f_{0}^{2}}{\omega_{0}^{2}}} \kappa_{n} \delta \kappa \\
& \times \oint \Xi(\phi) \mathcal{T}\left(\kappa_{n}, \phi\right) d \phi,
\end{aligned}
$$

where

$$
\delta \kappa \equiv \sqrt{\omega_{0}^{2}-f_{0}^{2}} \frac{\pi}{\bar{N} H},
$$


and $\kappa_{n}=n \delta \kappa$. The notation in (46) is suggested because $\delta \kappa$ is the spacing between the horizontal wavenumbers driven by the tidal forcing. The WKB approximation (45) indicates that the only properties of $N(z)$ which strongly affect tidal conversion are $N_{B}$ and $\bar{N}$.

\section{b. The limit of infinite depth, $H \rightarrow \infty$}

If $H \rightarrow \infty$, then normal modes are a needlessly cumbersome representation of the solution. The conversion rate $\mathcal{C}_{n}$ simplifies in this limit because $H$ appears in (45) only through $\delta \kappa \propto H^{-1}$ and $\kappa_{n}=n \delta \kappa$. When $\delta \kappa$ is small, the sum over normal modes, obtained by putting (45) into (29), can be approximated by an integral with $\kappa_{n}$ $\rightarrow \kappa$ and $\delta \kappa \rightarrow d \kappa$. Specifically,

$$
\begin{aligned}
\lim _{H \rightarrow \infty} C= & \frac{\rho_{0}}{8 \pi} N_{B}\left(U_{0}^{2}+V_{0}^{2}\right) \sqrt{1-\frac{f_{0}^{2}}{\omega_{0}^{2}}} \\
& \times \int_{0}^{\infty} \oint \Xi(\phi) \kappa \mathcal{T}(\kappa, \phi) d \phi d \kappa .
\end{aligned}
$$

We will give a detailed assessment of the accuracy of the integral approximation (47) in the next section. The main conclusion is that (47) approximates the sum in (29) provided that the horizontal scale of the topography is much less than $1 / \delta \kappa$.

In his equation (6) Bell (1975b) gives an expression for $C$ that is equivalent to (47). ${ }^{1}$ Bell uses the special coordinate system in which the axes of the tidal ellipse coincide with the coordinate axes. In our formulation the angle $\chi$ in (1) and (40) allows for a general orientation of the tidal ellipse relative to the coordinate system. To specialize (47) to the coordinate of the tidal ellipse notice that

$$
\kappa^{2}\left(U_{0}^{2}+V_{0}^{2}\right) \Xi=2 k^{\mathrm{T}} \mathbf{M k}
$$

where $\mathbf{k} \equiv[k, l]^{\mathrm{T}}=\kappa[\cos \phi, \sin \phi]^{\mathrm{T}}$ and $\mathbf{M}$ is the matrix

$$
\mathbf{M} \equiv\left[\begin{array}{cc}
U_{0}^{2} & U_{0} V_{0} \cos \chi \\
U_{0} V_{0} \cos \chi & V_{0}^{2}
\end{array}\right] .
$$

Since $\mathbf{M}$ is symmetric, it can be represented as

$$
\mathbf{M}=\mathbf{R J R}^{-1},
$$

where $\mathbf{R}$ the rotation matrix whose columns are the eigenvectors of $\mathbf{M}$ and $\mathbf{J}$ is the diagonal matrix

$$
\mathbf{J}=\left[\begin{array}{cc}
U_{+}^{2} & 0 \\
0 & U_{-}^{2}
\end{array}\right]
$$

with

\footnotetext{
${ }^{1}$ Bell refers to his $P(\kappa, \lambda)$ as the "spectrum of the bottom topography," whereas actually $P=|\tilde{h}|^{2}$-see the discussion surrounding (41). Because of (14), Bell's parameter $\beta$ is small. Thus Bell's sums over $n$ are simplified by keeping only the first term and using $J_{1}(\beta)$ $\approx \beta / 2$.
}

$$
U_{ \pm}^{2}=\frac{1}{2}\left(U_{0}^{2}+V_{0}^{2} \pm \sqrt{\left(U_{0}^{2}+V_{0}^{2}\right)^{2}+4 U_{0}^{2} V_{0}^{2} \cos \chi}\right) .
$$

The diagonal entries of $\mathbf{J}$ are the eigenvalues of $\mathbf{M}$. Equivalently, $U_{+}$and $U_{-}$are the major and minor axes of the tidal ellipse.

Now change the variables of integration in (47) to

$$
\mathbf{k}^{\prime}=\mathbf{R}^{-1} \mathbf{k}
$$

Since $\mathbf{R}$ is a rotation matrix, the Jacobian of the transformation $\mathbf{k} \rightarrow \mathbf{k}^{\prime}$ is unity and $\kappa=\sqrt{k^{\prime 2}+l^{\prime 2}}$. In terms of $\mathbf{k}^{\prime}=\left[k^{\prime}, l^{\prime}\right]^{\mathrm{T}}$, the integral on the right-hand side of (47) becomes

$$
\begin{aligned}
C= & \frac{\rho_{0}}{8 \pi^{2}} N_{B} \sqrt{1-\frac{f_{0}^{2}}{\omega_{0}^{2}}} \\
& \times \int_{-\infty}^{\infty} \int_{-\infty}^{\infty} \frac{U_{+}^{2} k^{\prime 2}+U_{-}^{2} l^{\prime 2}}{\sqrt{k^{\prime 2}+l^{\prime 2}}}\left|\tilde{h}\left(k^{\prime}, l^{\prime}\right)\right|^{2} d k^{\prime} d l^{\prime},
\end{aligned}
$$

where (38) has been used to replace $\mathcal{T}$ in favor of $|\tilde{h}|^{2}$. Equation (54) is equivalent to Eq. (6) in Bell (1975b).

\section{c. The one-dimensional limit}

Starting from the WKB approximation in (45) we can also take the limit in which the topography becomes almost one-dimensional. That is, suppose that

$$
h(x, y)=F_{L}(y) h_{1}(x)
$$

where $F_{L}(y)=1$ if $-L / 2<y<L / 2$ and $F_{L}(y)=0$ otherwise. The function $h_{1}(x)$ allows for arbitrary variation in $x$ of the topography. As $L \rightarrow \infty$, the solution will reduce to the one-dimensional special case. In this limit the main contribution to the $\phi$ integral in (45) comes from the neighborhood of $\phi=0$ and $\phi=\pi$, where

$$
\begin{aligned}
\Xi & \approx \frac{2 U_{0}^{2}}{U_{0}^{2}+V_{0}^{2}}, \\
\mathcal{T}\left(\kappa_{n}, \phi\right) & \approx \frac{2}{\pi} l^{-2} \sin ^{2}\left(\frac{l L}{2}\right) \kappa_{n} \tilde{h}_{1}\left(\kappa_{n}\right) \tilde{h}_{1}^{*}\left(\kappa_{n}\right) .
\end{aligned}
$$

Evaluating the simplified integrals gives the leading order approximation

$$
C_{n} \approx \rho_{0} L N_{B} U_{0}^{2} \sqrt{1-\frac{f_{0}^{2}}{\omega_{0}^{2}}} \kappa_{n} \tilde{h}_{1}\left(\kappa_{n}\right) \tilde{h}_{1}^{*}\left(\kappa_{n}\right) \frac{\delta \kappa}{2 \pi} .
$$

We have verified that (57) is also obtained if one makes the ab initio idealization that the topography depends only on $x$ and proceeds directly from (25).

\section{d. Recovery of Bell's formula}

Bell's (1975a) result in (2) assumes that $H \rightarrow \infty$ and that the topography varies only in $x$. We recover this 


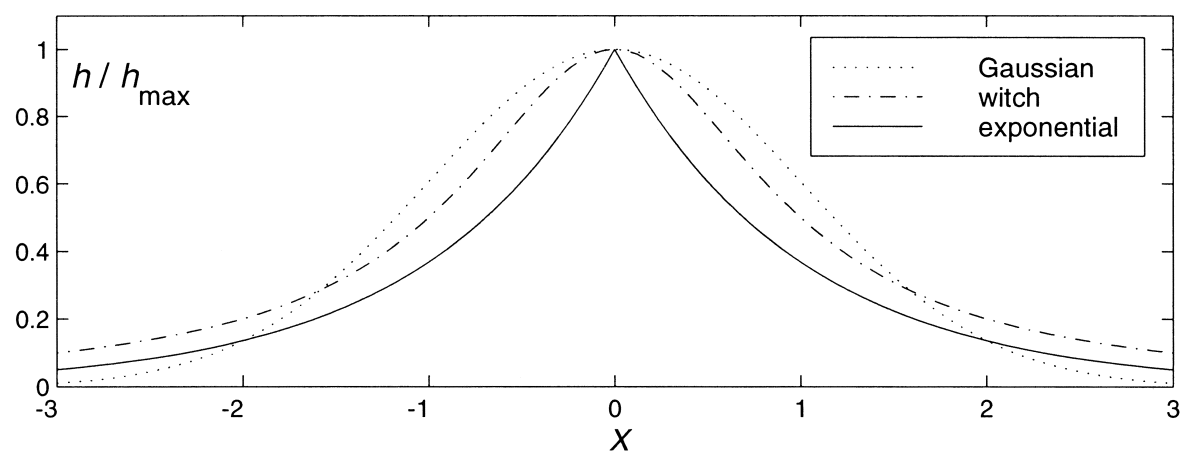

FIG. 2. Three model topographic profiles. The exponential model, $h \propto \exp (-|x| / l)$, has a discontinuous derivative at $X \equiv x / l=0$.

doubly special case by starting with either (47) or (57) and making the appropriate reductions. The easiest path starts by putting (57) into the modal sum (29) and then taking $H \rightarrow \infty$ (equivalently $\delta \kappa \rightarrow 0$ ) to obtain an integral over $\kappa$.

\section{Tidal conversion by a ridge}

In this section we use (57) to estimate the tidal conversion by a one-dimensional ridge in an ocean of finite depth $H$. We make comparisons between the three topographic profiles illustrated in Fig. 2. All of the profiles are characterized by a maximum height $h_{\max }$ and a horizontal scale $l=1 / \mu$.

\section{a. Summary}

We write the conversion rate of a ridge, obtained from the modal sum in (29) and the WKB approximation to $C_{n}$ in (57), as

$$
C=\mathcal{C}_{\mathrm{Bell}} S\left(\frac{\bar{N} H}{\pi l \sqrt{\omega_{0}^{2}-f_{0}^{2}}}\right) .
$$

In (58),

$$
\mathcal{C}_{\text {Bell }}=\mathcal{D} L \rho_{0} U_{0}^{2} N_{B} \sqrt{1-\frac{f_{0}^{2}}{\omega_{0}^{2}}} h_{\max }^{2},
$$

where $\mathcal{D}$ is a numerical constant that depends only on the profile (see the third column of Table 1). $\mathcal{C}_{\text {Bell }}$, originally defined in (2), is the conversion rate with $H=$ $\infty$. The reduction factor $S(\alpha)$ in (58) increases monotonically from $S(0)=0$ to $S(\infty)=1$. Explicit expres- sions for $S(\alpha)$ are given in the final column of Table 1 and these three examples are graphed in Fig. 3.

\section{b. Discussion of the tidal conversion by a finite-depth ridge}

Both $l$ and $H$ appear in (58) via the nondimensional combination

$$
\alpha \equiv \frac{\bar{N} H \mu}{\pi \sqrt{\omega_{0}^{2}-f_{0}^{2}}}=\frac{1}{l \delta \kappa} .
$$

The discussion surrounding (47) emphasizes that, as $H$ $\rightarrow \infty$, the sum over discrete vertical normal modes can be approximated by a modal continuum. The function $S(\alpha)$ provides a quantitative indication of the accuracy or rate of convergence of this approximation. Specifically, if $\alpha=2$ then $C_{\text {Bell }}$ is within $10 \%$ of $C$ for all profiles in Fig. 2. Thus "large $\alpha$ " means $\alpha \gtrsim 2$.

Because $\alpha$ is an important nondimensional parameter we offer several physical interpretations. The most fundamental is suggested by $\delta \kappa=1 /(\alpha l)$ : the interval between the horizontal wavenumbers excited by the tidal forcing is controlled by $\alpha$ and the topographic length scale $l$. The limit of a modal continuum is approached by making $\alpha \propto H$ large, or alternatively by reducing the horizontal scale $l$ of the topography. We illustrate this point by calculating the fraction of the total conversion $C$ contained in the first $n$ vertical modes:

$$
F_{n}(\alpha) \equiv \sum_{m=1}^{n} \mathcal{C}_{m} / \sum_{m=1}^{\infty} \mathcal{C}_{m} .
$$

Figure 4 shows $F_{n}(\alpha)$ for the witch profile; there is

TABLE 1. Results for three model topographies: the Gaussian, the witch, and (in the final row) the exponential. We use the notation $X=$ $x / l, K=k l$, and $n^{\prime}=n / \alpha$. The nondimensional constant $\mathcal{D}$ appears in (59) and the sum $S(\alpha)$ is the reduction factor in (58).

\begin{tabular}{cccc}
\hline \hline$h(x) / h_{\max }$ & $\tilde{h}(k) / h_{\max }$ & $\mathcal{D}$ & $S(\alpha)$ \\
\hline $\exp \left(-X^{2} / 2\right)$ & $\sqrt{2 \pi} 1 \exp \left(-K^{2} / 2\right)$ & $1 / 2$ & $2 \alpha^{-1} \sum_{n=1}^{\infty} n^{\prime} \exp \left(-n^{\prime 2}\right)$ \\
$\left(1+X^{2}\right)^{-1}$ & $\pi 1 \exp (-|K|)$ & $\pi / 8$ & $4 \alpha^{-1} \sum_{n=1}^{\infty} n^{\prime} \exp \left(-2 n^{\prime}\right)$ \\
$\exp (-|X|)$ & $2 l /\left(1+K^{2}\right)$ & $\pi^{-1}$ & $2 \alpha^{-1} \sum_{n=1}^{\infty} n^{\prime} /\left(1+n^{\prime 2}\right)^{2}$ \\
\hline
\end{tabular}



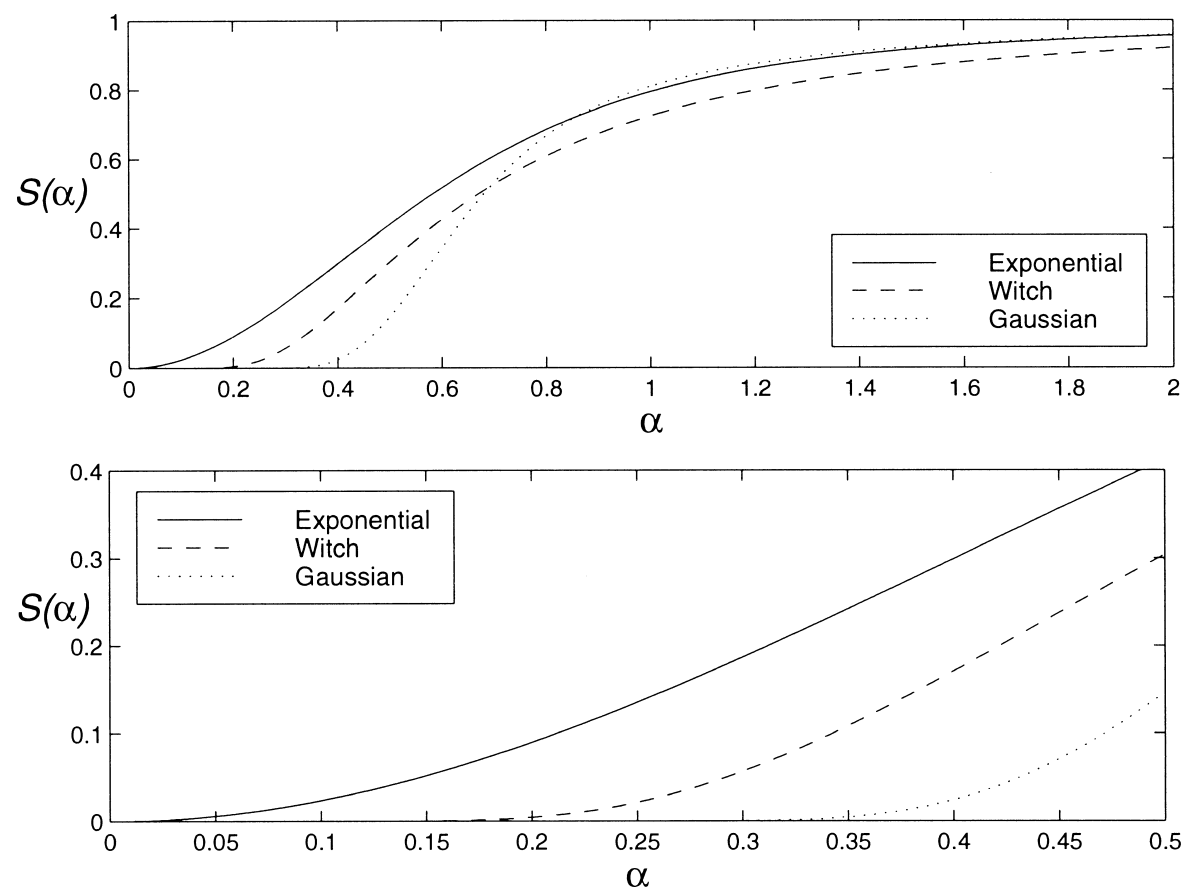

FIG. 3. The sum $S(\alpha)$ in (58) for the witch of Agnesi, the exponential and Gaussian profiles. The analytic expressions for $S(\alpha)$ are in the final column of Table 1. (top) The approach to the $H \rightarrow \infty$ limit, $S(\infty)=1$; (bottom) $S(\alpha)$ with $0<\alpha<1 / 2$.

significant projection into the vertical modes with $n \geq$ 3 only if $\alpha$ is greater than about 2 .

To roughly estimate the size of $\alpha$, notice that a ridge of width $l=\mu^{-1}$ will force internal tides with horizontal wavenumbers $k \sim \mu$. Then, using the internal wave dispersion relation, we can interpret $\alpha$ as the ratio

$$
\alpha \sim \frac{\text { ocean depth }}{\text { vertical wavelength of the internal tide }} .
$$

Since the deformation radius of the first mode is $\lambda_{1} \approx$ $\bar{N} H / \pi f_{0}$, we can also write

$$
\alpha=\frac{1}{\sqrt{\left(\omega_{0} / f_{0}\right)^{2}-1}} \frac{\lambda_{1}}{l} .
$$

With a typical midlatitude deformation radius of $\lambda_{1}=$ $30 \mathrm{~km}$, and $\omega_{0}=2 f_{0}$, we have $\alpha \sim 17 / l$ where the ridge width $l$ is in units of kilometers. Thus, from Fig. 3 , a ridge of width $17 \mathrm{~km}$ will have a reduction factor of $S(1) \sim 0.8$. With $\alpha=1$ the reduction factor is insensitive to changes in the topographic profile. However, if the ridge width is doubled to $34 \mathrm{~km}$, then $\alpha \sim 1 / 2$ and the details of the topographic profile make large changes in $S$ (see the bottom panel of Fig. 3). The main point is that the rise of $S$ at small $\alpha$ is very sensitive to the high wavenumber structure of $\tilde{h}(k)$ : the profiles with rapid decay of $\tilde{h}(k)$ at large $k$ (the Gaussian and the witch) have very small reduction factors if $\alpha<0.4$.

\section{c. The radiated waves}

To visualize the solution we need an explicit solution of (33). The exponential topography in the final row of Table 1 is convenient because the solution is

$$
p_{n}(x)=\frac{h(x)}{\mu^{2}+\kappa_{n}^{2}}-\frac{i}{\kappa_{n}} \frac{\mu h_{\max }}{\mu^{2}+\kappa_{n}^{2}} e^{i \kappa_{n}|x|} .
$$

The first term on the right-hand side of (64) is a forced response trapped over the ridge and the second term is the radiated wave. Figure 5 shows the buoyancy field for $\alpha=0.1$ and $\alpha=1$. As $\alpha$ increases for fixed $H$ and $l$, the horizontal scale of the internal tide increases.

\section{Gaussian topography}

The Gaussian seamount

$$
h(x, y)=h_{\max } e^{-\left(x^{2}+y^{2}\right) / 2 a^{2}}
$$

is a simple example of a two-dimensional topography. In the case of a very deep ocean we can use the WKB formula in (47) to calculate the conversion rate. The result is

$$
C_{\text {axi }}=\frac{1}{8} \pi^{3 / 2} \rho_{0} N_{B} a\left(U_{0}^{2}+V_{0}^{2}\right) \sqrt{1-\frac{f_{0}^{2}}{\omega_{0}^{2}}} h_{\max }^{2} .
$$

In the conclusion we use (66) to estimate the conversion produced by the global inventory of over 1.4 million seamounts. 


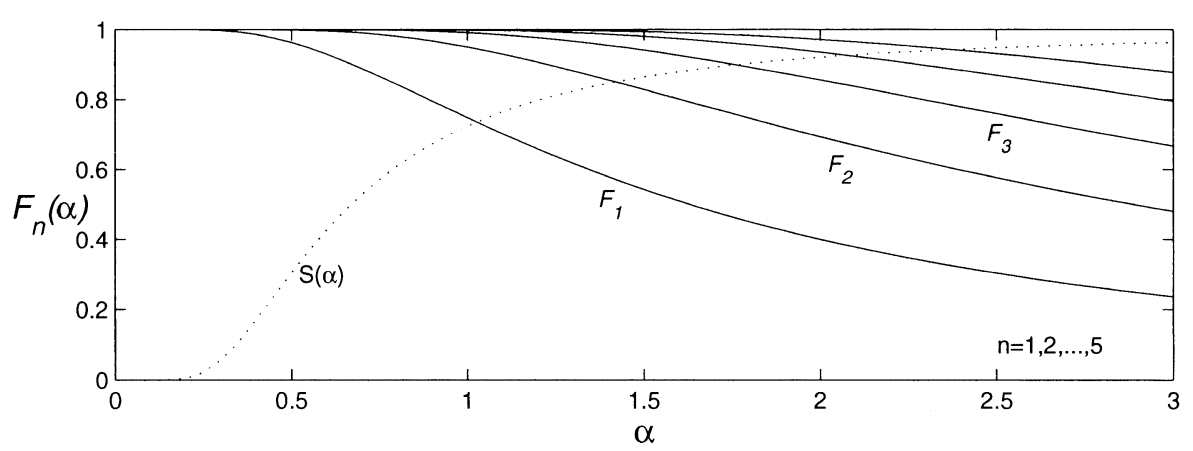

FIG. 4. $F_{n}(\alpha)$, defined in (61) for the witch profile; $F_{n}$ is the fraction of converted energy in the first $n$ vertical modes. When $\alpha<1$ the first two vertical modes contain nearly all of the converted tidal energy.

\section{a. Anisotropic topography}

We now move to the case of general two-dimensional Gaussian topography, given by

$$
h(x, y)=h_{\max } e^{-\mu^{2} x^{2} / 2-\nu^{2} y^{2} / 2} .
$$

We have aligned the topography with the $x$ and $y$ axes; an arbitrary angle between the tidal ellipse and the major axis of the topography can be obtained by adjusting the phase $\chi$ in (1). We characterize the scale of the topography using the equivalent radius

$$
a \equiv(\mu \nu)^{-1 / 2}
$$

and the anisotropy with

$$
\beta \equiv \nu / \mu \text {. }
$$

Then, again using the WKB approximation, the total conversion rate is, from (29) and (45),

$$
C=\frac{1}{2} \pi \rho_{0} N_{B}\left(U_{0}^{2}+V_{0}^{2}\right) \sqrt{1-\frac{f_{0}^{2}}{\omega_{0}^{2}}} h_{\max }^{2} a \sum_{n=1}^{\infty} \frac{n^{2}}{\alpha^{3}} e^{-\lambda_{n}^{+}}\left[I_{0}\left(\lambda_{n}^{-}\right)+\gamma I_{1}\left(\lambda_{n}^{-}\right)\right],
$$

where $I_{m}$ is a modified Bessel function. In (70), and throughout this section, we change the definition of $\alpha$ to

$$
\alpha \equiv \frac{\bar{N} H}{\pi a \sqrt{\omega_{0}^{2}-f_{0}^{2}}}=\frac{1}{a \delta \kappa},
$$

where $a$ is the equivalent radius defined in (68). Additionally in (70)

$$
\lambda_{n}^{ \pm} \equiv \frac{1}{2} n^{2} \frac{\left(\beta^{-1} \pm \beta\right)}{\alpha^{2}}, \quad \gamma \equiv \frac{\left(U_{0}^{2}-V_{0}^{2}\right)}{\left(U_{0}^{2}+V_{0}^{2}\right)} .
$$

Using $C_{\text {axi }}$ in (66) we rewrite (70) as

$$
C=C_{\text {axi }}\left[R_{0}(\alpha, \beta)+\gamma R_{1}(\alpha, \beta)\right]
$$

where, with $m=0$ and 1 ,

$$
R_{m}(\alpha, \beta) \equiv \frac{4}{\alpha^{3} \sqrt{\pi}} \sum_{n=1}^{\infty} n^{2} e^{-\lambda_{n}^{+}} I_{m}\left(\lambda_{n}^{-}\right) .
$$

The square bracket on the right-hand side of (73) is the ratio of the conversion $C$ to the conversion produced by an equivalent axisymmetric seamount in an ocean with $H=\infty$. This ratio depends on the three nondimensional parameters $\alpha, \beta$, and $\gamma$.
From the definitions of $\lambda_{n}^{ \pm}$in (72) and the parity of $I_{m}$ it follows that

$$
R_{m}(\alpha, 1 / \beta)=(-1)^{m} R_{m}(\alpha, \beta)
$$

In physical terms, the identity above arises because the correspondence of the $x$ direction to the major or minor axis of the elliptical topography is an accident of notation. Equation (75) is useful because one can simplify the task of calculating and plotting $R_{m}$ by limiting attention to $\beta \leq 1$.

\section{b. The infinite depth limit, $\alpha=\infty$}

The large- $\alpha$ limits of $R_{0}$ and $R_{1}$, corresponding to an infinitely deep ocean, are obtained once again by converting the sums in (74) into integrals over $\kappa=n /(\alpha a)$. Putting the integral representation of the modified Bessel function $I_{m}$ [see formula 8.431.5 in Gradshteyn and Ryzhik (2000, p. 907)] into this expression, and then reversing the order of integration leads to simplification. The result is 

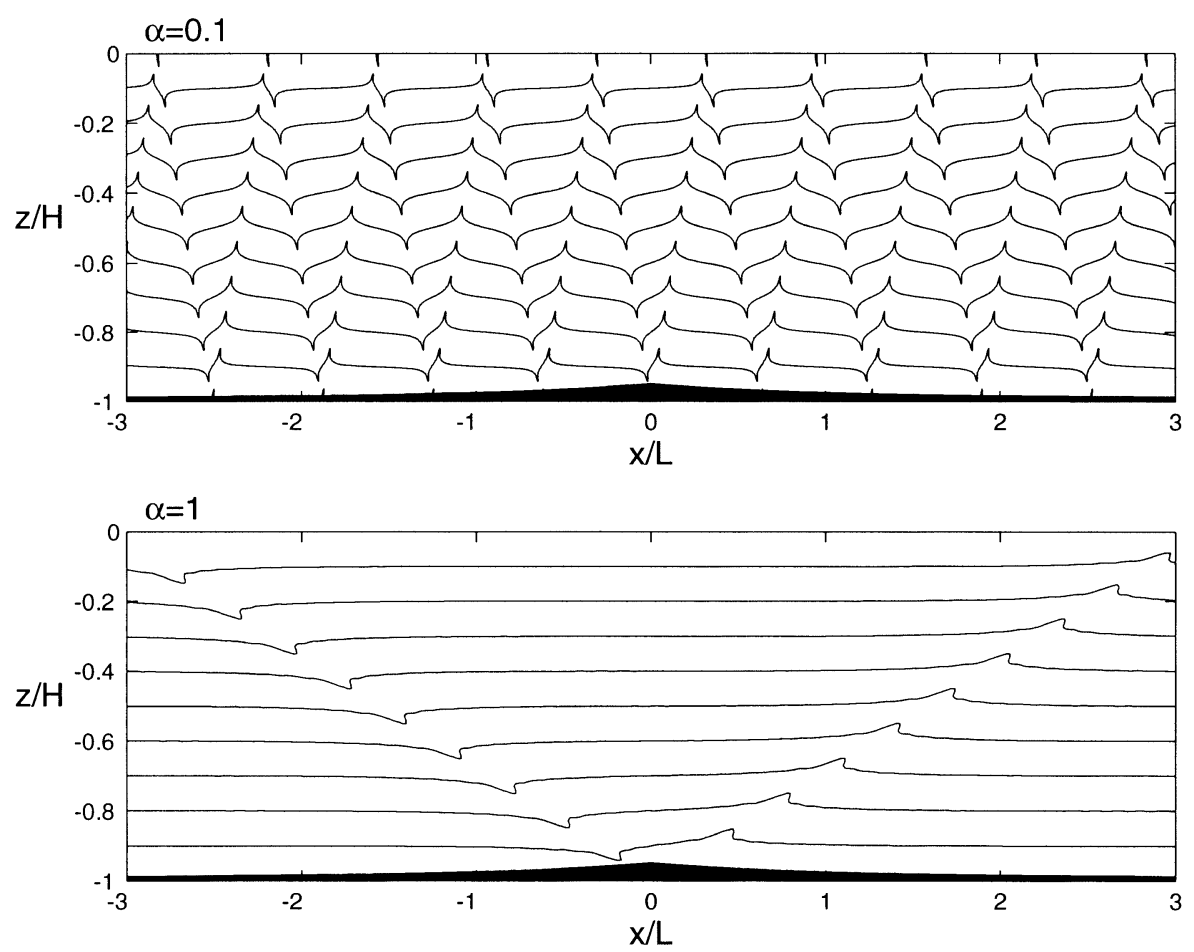

FIG. 5. Buoyancy field for the exponential profile in the final row of Table 1: (top) $\alpha=0.1$ and (bottom) $\alpha=1$. When $\alpha$ is small the surface reflected waves interact with topography and the resulting destructive interference reduces the conversion rate. This is a linear solution and the arbitrary amplitude of $b$ is large in order to make the radiating internal tide visible.

$$
\begin{aligned}
R_{0}(\infty, \beta)=\frac{2}{\pi \sqrt{\beta}} E\left(\sqrt{1-\beta^{2}}\right) \quad \text { and } \\
R_{1}(\infty, \beta)=\frac{2}{\pi \sqrt{\beta}}\left[\frac{1+\beta^{2}}{1-\beta^{2}} E\left(\sqrt{1-\beta^{2}}\right)\right. \\
\left.\quad-\frac{2 \beta^{2}}{1-\beta^{2}} K\left(\sqrt{1-\beta^{2}}\right)\right],
\end{aligned}
$$

where $K(k)$ and $E(k)$ are the complete elliptic integrals of the first and second kinds, respectively, with parameter $k=\sqrt{1-\beta^{2}}$. (For definitions of $K$ and $E$, see section 8.11 of Gradshteyn and Ryzhik; notice that Gradshteyn and Ryzhik's $k^{\prime} \equiv \sqrt{1-k^{2}}$ is equal to our $\beta$.) The functions $R_{0}(\infty, \beta)$ and $R_{1}(\infty, \beta)$ are graphed in Fig. 6.

As an example, we follow Holloway and Merrifield (1999) and consider the conversion produced by an elongated Gaussian with minor $(x)$ axis $\mu^{-1}=16 \mathrm{~km}$ and major $(y)$ axis $3 \mu^{-1}$. In other words, $\beta=1 / 3$ and the equivalent radius is $a=\sqrt{3} \times 16 \approx 27.7 \mathrm{~km}$. From (76) and (77) we compute $R_{0}(\infty, 1 / 3)=1.23$ and $R_{1}(\infty$, $1 / 3)=0.84$. Thus the conversion of the elongated topography is between $R_{0}-R_{1}=0.39$ and $R_{0}+R_{1}=$ 2.07 that of the equivalent axisymmetric topography. The smaller figure applies if the tide is linearly polarized along the major axis of the topography $\left(U_{0}=0\right.$ and $\gamma$ $=-1$ ) while the larger number applies if the tide is linearly polarized along minor axis $\left(V_{0}=0\right.$ and $\gamma=$ 1). Notice that Holloway and Merrifield compare the conversion of the elongated topography to that of a circular seamount with a radius equal to the minor axis of the ellipse, that is, a seamount with radius $a^{\prime}=16 \mathrm{~km}$. In these terms the conversion is between 0.68 and 3.58 times that of the "minor seamount."

Figure 7 shows $\mathcal{C}(\infty, \beta, \gamma) / \mathcal{C}_{\text {axi }}$ (this ratio corresponds to $\mathcal{D}$ of the previous section). The conversion rate drops to zero as $\gamma \rightarrow-1$ and $\beta \rightarrow 0$; this is a meriodionally oriented ridge with a linearly polarized meridional tide. The conversion rate increases to its maximum value for $\gamma=-1$ as $\beta$ increases, which corresponds to the onedimensional problem of the previous section. The symmetry about the origin corresponds to $x \leftrightarrow y, U_{0} \leftrightarrow V_{0}$, and $\mu \leftrightarrow \nu$.

\section{c. Finite-depth effects}

The approach of $C$ to its limiting value as $\alpha \rightarrow \infty$ is very similar to that exhibited by the functions $S(\alpha)$ of the previous section. The behavior of $R_{0}$ and $R_{0}-R_{1}$ (the results for $R_{0}+R_{1}$ are the same under $\beta \leftrightarrow \beta^{-1}$ ) is shown in Fig. 8. These two curves show the generic behavior of $C=C_{\mathrm{axi}}\left(R_{0}+\gamma R_{1}\right)$, but the prefactor has been left out to show the different limiting values depending on the value of $\beta$.

For large infinite-depth conversion rates (e.g., large 

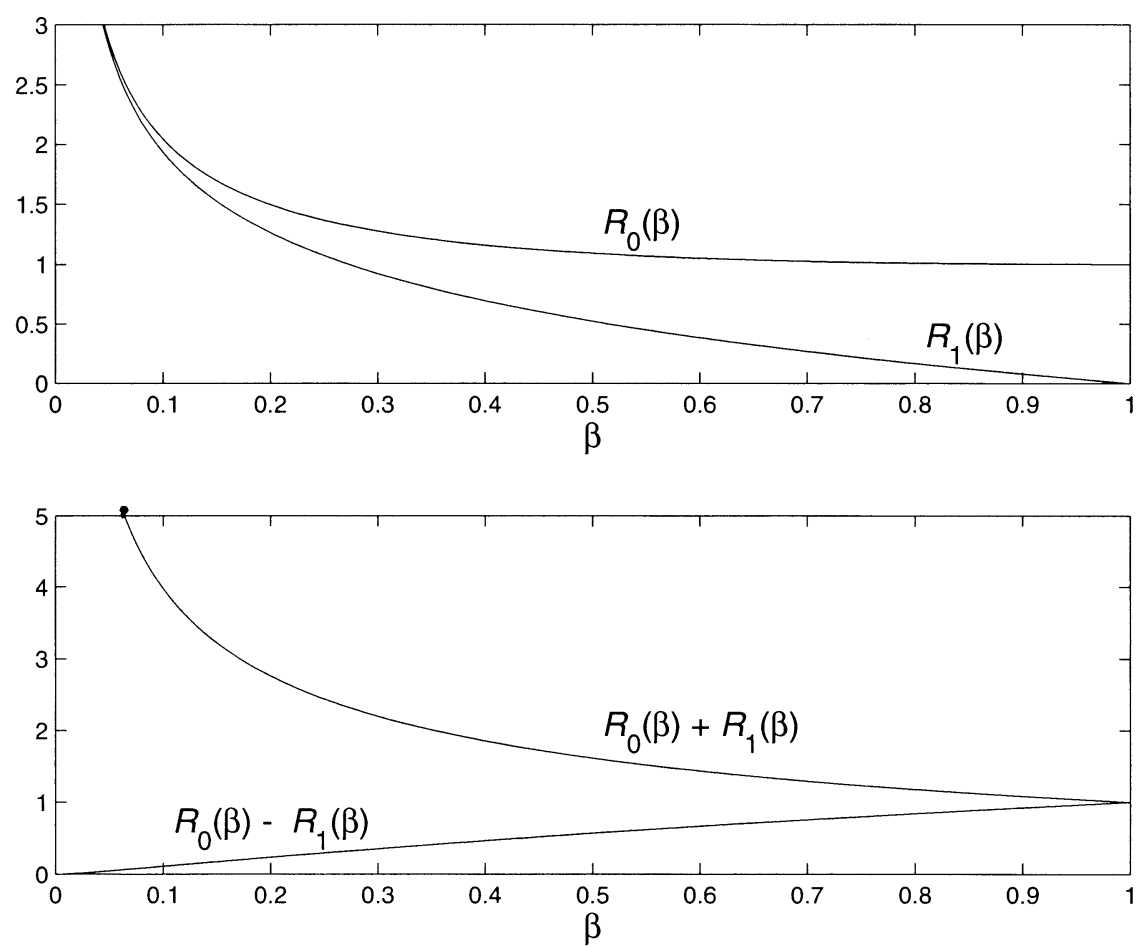

FIG. 6. (top) The functions $R_{0}(\infty, \beta)$ and $R_{1}(\infty, \beta)$ in (76) and (77); (bottom) the linear combination $R_{0}+\gamma R_{1}$ in (73) lies between $R_{0}-R_{1}$ and $R_{0}+R_{1}$.

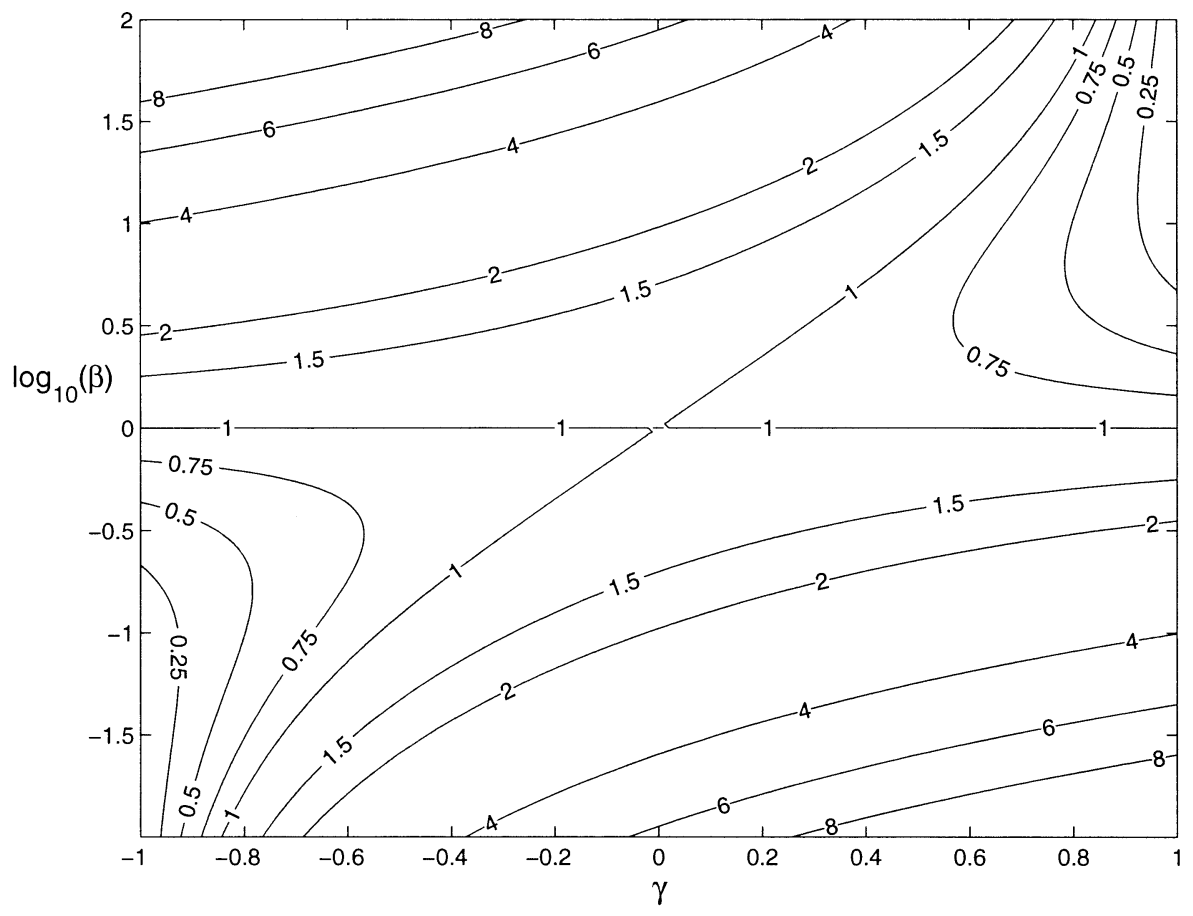

FIG. 7. The $\alpha=\infty$ limit of $d C_{\text {axi }}$ computed using (76) and (77). 

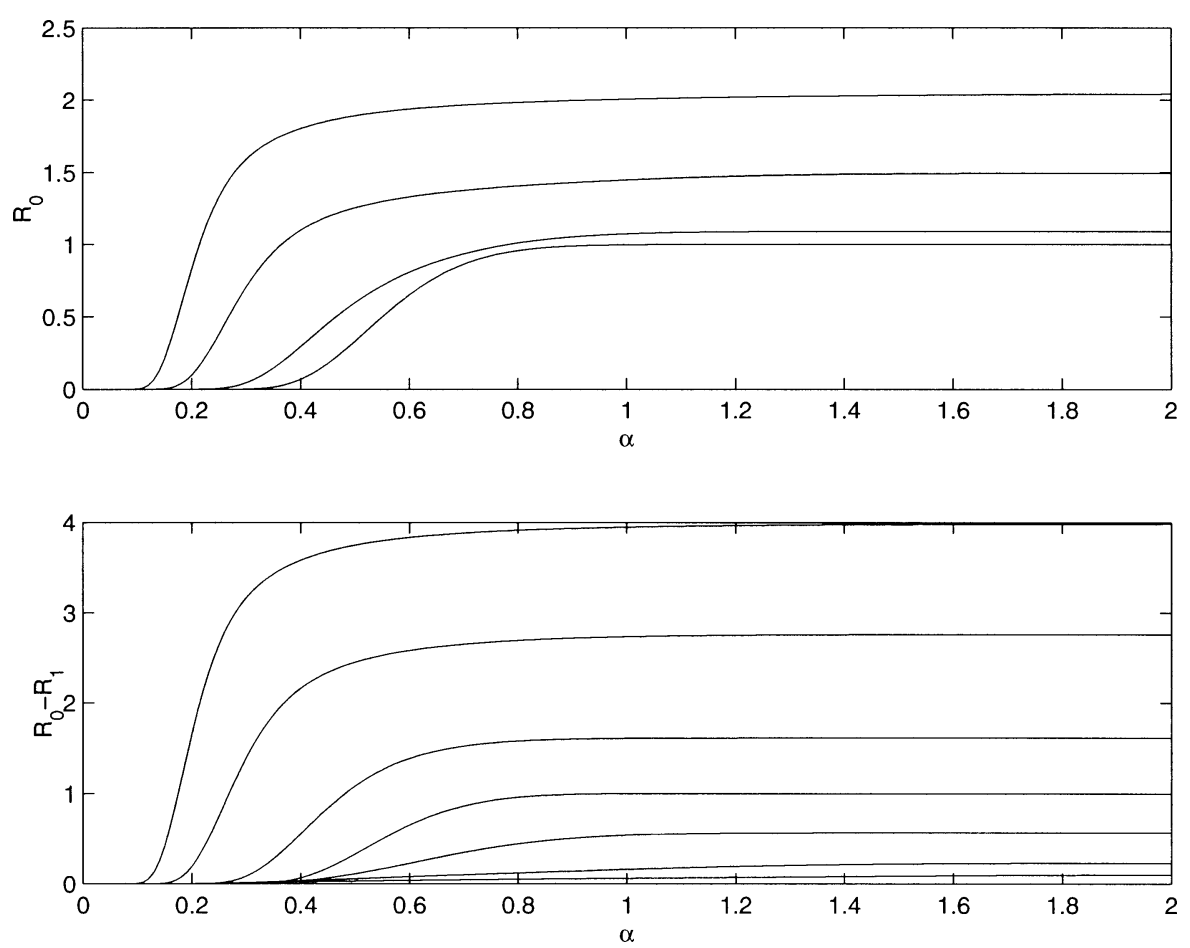

FIG. 8. Behavior of $R_{0}$ and $R_{0}-R_{1}$ as functions of $\alpha$ for $\beta=0.1,0.2,0.5,1,2,5$, and 10. (top) The curves for the $\beta$ and $\beta^{-1}$ are the same, and the curve with the smallest limit as $\alpha \rightarrow \infty$ (namely, 1) corresponds to $\beta=1$; (bottom) the limiting value of $R_{0}-R_{1}$ increases as $\beta$ decreases.

$\beta$ and $\gamma=-1$ ), the growth with $\alpha$ is rapid. Conversely, for small infinite-depth conversion rates, the growth is slow. The overall behavior is similar to that for onedimensional topography except in cases of weak conversion, when the tide is aligned with the major axis of the topography.

\section{Conclusions}

The analytic estimates of conversion in this paper are hostage to the small-topography assumption in (15). This parametric restriction enables us to apply the bottom boundary condition at a flat surface and so obtain a tractable problem. Recent results of Balmforth et al. (2001, submitted to J. Phys. Oceanogr.) indicate that the small-topography approximation gives only a slight underestimate of the conversion, even if the topographic slopes approach the critical condition. For example, Balmforth et al. consider a Gaussian ridge with critical slope and $H=\infty$. In this case the true conversion is only $14 \%$ greater than one would estimate using the small-topography approximation. These results give us some confidence that the small-topography approximation is quantitatively accurate provided that the topographic slope is modestly less than the ray slopes. Encouraged by these results we conclude by using the expression for the conversion of an axisymmetric seamount in (66) to estimate the global conversion produced by seamounts.
Jordan et al. (1983) and Smith and Jordan (1988) constructed a parameterized model of seamount statistics in the Pacific. We adapt their model and use (66) to make a rough estimate of the tidal conversion produced by an ensemble of seamounts. According to Jordan et al., seamounts cover about $6 \%$ of the seafloor and the density of seamounts is $(4 \pm 1.1) \times 10^{-9} \mathrm{~m}^{-2}$. In other words, there are about 4000 seamounts per $10^{6}$ $\mathrm{km}^{2}$. The radii of the seamount population has an exponential probability density function

$$
\mathcal{P}(a)=\bar{a}^{-1} \exp (-a / \bar{a})
$$

where $\bar{a} \approx 1.6 \mathrm{~km}$ is the average radius of the population. As a rule of thumb, the height and radius of a seamount are related by $h_{\max } \approx a / 5$.

Using the numbers above we consider an "average" seamount with a radius of $1.6 \mathrm{~km}$ and a height of 320 $\mathrm{m}$. To use (66) we further suppose that $f_{0}=10^{-4} \mathrm{~s}^{-1}$, $\omega_{0}=2 f_{0}$ and that the abyssal buoyancy frequency is $N_{B}=5 f_{0}$ (that is, $0.29 \mathrm{cph}$ ). For the tidal currents we take $U_{0}=V_{0}=10^{-2} \mathrm{~m} \mathrm{~s}^{-1}$. Putting these numbers into (66) we get for the average seamount

$$
\bar{C}_{\text {axi }} \approx 10^{4} \mathrm{~W} \text {. }
$$

In other words, the average seamount produces about 100 lightbulbs of energy.

To calculate the total tidal conversion of the seamount population we use the rule of thumb suggested by Jordan et al. (1983) that $h_{\max } \approx a / 5$ so that $C_{\mathrm{axi}} \propto a^{3}$. This means 
that the larger seamounts make a greater contribution to the conversion. Indeed, since the function $x^{3} \exp (-x)$ peaks at $x=3$, it is the seamounts with $a=3 \bar{a}$ that make the largest contribution to the total conversion. And, since

$$
\int_{0}^{\infty} a^{3} \mathcal{P}(a) d a=6 \bar{a}^{3}
$$

the total conversion of the population is actually $6 \mathcal{N} \bar{C}_{\text {axi }}$, where $\mathcal{N}$ is the total number of seamounts. Considering a square of seafloor $1000 \mathrm{~km}$ on a side (containing $\mathcal{N}$ $=4000$ seamounts), the total conversion is then

$$
C_{\mathrm{sqr}} \approx \frac{1}{4} \mathrm{GW} \text {. }
$$

This is small, but not entirely negligible. For instance, using altimetry Ray and Mitchum (1996) estimate 15 GW of conversion into the first baroclinic mode by the Hawaiian Ridge. And Munk (1997) estimated that 50 $000 \mathrm{~km}$ of submarine ridges produce $200 \mathrm{GW}$ of $M_{2}$ conversion. Thus it takes 60 of these $1000 \mathrm{~km} \times 1000$ $\mathrm{km}$ squares to equal Ray and Mitchum's estimate for Hawaii. But there are 360 squares paving the global seafloor. Thus a blithe extrapolation of (81) would indicate a seamount conversion equivalent to 6 of Ray and Mitchum's Hawaiis or $45 \%$ of Munk's submarine ridges. This implies that rather small topographic features might make a palpable contribution to the total abyssal conversion. [Notice that $3 \bar{a}=4.8 \mathrm{~km}$ is below the resolution of Smith and Sandwell's (1997) satellite altimetry.]

Because of the 5-km scale of these seamounts finitedepth corrections are unimportant. That is, $\alpha$ in (71) is large. We can also make an a posteriori assessment of the small-topography approximation. A Gaussian seamount with radius $a$ and height $h_{\max }=a / 5$ has a maximum slope $h_{\max } / \sqrt{e} a \sim 0.12$. On the other hand, the slope of a radiated tidal beam is $\sqrt{\omega_{0}^{2}-f_{0}^{2}} / N \sim 0.35$. The ratio of topographic slope to ray slope is thus $1 / 3$, which is small enough that the assumption of weak topography is good.

Acknowledgments. We thank Chris Garrett and Lou St. Laurent for useful advice during the early stages of this work. WRY is supported by NSF OCE96-16017.
SGLS acknowledges a UCSD 2001 Chancellor's Summer Faculty Fellowship. The order of authorship is alphabetical.

\section{REFERENCES}

Baines, P. G., 1973: The generation of internal tides by flat-bump topography. Deep-Sea Res., 20, 179-205.

Bell, T. H., 1975a: Lee waves in stratified fluid with simple harmonic time dependence. J. Fluid Mech., 67, 705-722.

- 1975b: Topographically generated internal waves in the open ocean. J. Geophys. Res., 80, 320-327.

Cox, C., and H. Sandstrom, 1962: Coupling of internal and surface waves in water of variable depth. J. Oceanogr. Soc. Japan, 18 (20th anniversary volume), 499-513.

Cummins, P. F., J. Y. Cherniawsky, and M. G. G. Foreman, 2001: North Pacific internal tides from the Aleutian Ridge: Altimeter observations and modeling. J. Mar. Res., 59, 167-191.

Egbert, G. D., and R. D. Ray, 2000: Significant dissipation of tidal energy in the deep ocean inferred from satellite altimeter data. Nature, 405, 775-778.

Gradshteyn, I. S., and I. M. Ryzhik, 2000: Table of Integrals, Series and Products. 6th ed. Academic Press, $1163+$ xlvii pp.

Holloway, P. E., and M. A. Merrifield, 1999: Internal tide generation by seamounts, ridges and islands. J. Geophys. Res., 104 (C11), 25 937-25 951 .

Jordan, T. H., W. W. Menard, and D. K. Smith, 1983: Density and size distribution of seamounts in the eastern Pacific inferred from wide-beam sounding data. J. Geophys. Res., 88 (B12), 10508 10518.

Kang, S. K., M. G. G. Foreman, W. R. Crawford, and J. Y. Cherniawsky, 2000: Numerical modelling of internal tide generation along the Hawaiian Ridge. J. Phys. Oceanogr., 30, 1083-1098.

Ledwell, J. R., E. T. Montgomery, K. L. Polzin, L. C. St. Laurent, R. W. Schmitt, and J. M. Toole, 2000: Evidence of enhanced

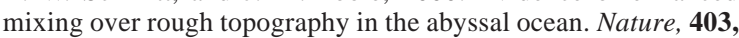
179-182.

Li, M., 2001: Energetics of internal tides radiated from deep-ocean topographic features. J. Mar. Res.,

Munk, W. H., 1997: Once again: Once again—tidal friction, 1997. Progress in Oceanography, Vol. 40, Pergamon, 7-35.

_, and C. I. Wunsch 1998: Abyssal recipes. II: Energetics of tidal and wind mixing. Deep-Sea Res., 45, 1977-2010.

Rattray, M., 1960: On the coastal generation of internal tides. Tellus, 12, 54-61.

Ray, R. D., and G. T. Mitchum, 1996: Surface manifestation of internal tides generated near Hawaii. Geophys. Res. Lett., 23, 2101-2104.

Smith, D. K., and T. H. Jordan, 1988: Seamount statistics in the Pacific Ocean. J. Geophys. Res., 93 (B4), 2899-2918.

Smith, W. H. F., and D. T. Sandwell, 1997: Global sea floor topography from satellite altimetry and ship depth soundings. Science, 277, $1956-1962$.

Wunsch, C., 1975: Internal tides in the ocean. Rev. Geophys., 13, 167-182. 